\title{
BMJ Open Prehospital triage of patients with suspected stroke symptoms (PRESTO): protocol of a prospective observational study
}

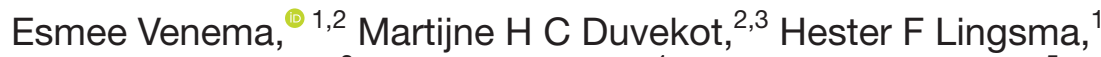 \\ Anouk D Rozeman, ${ }^{3}$ Walid Moudrous, ${ }^{4}$ Frederique H Vermeij, ${ }^{5}$ Marileen Biekart, ${ }^{6}$ \\ Aad van der Lugt, ${ }^{7}$ Henk Kerkhoff, ${ }^{3}$ Diederik W J Dippel, ${ }^{2}$ Bob Roozenbeek, ${ }^{2,7}$ on \\ behalf of the PRESTO investigators
}

To cite: Venema E, Duvekot MHC, Lingsma HF, et al. Prehospital triage of patients with suspected stroke symptoms (PRESTO): protocol of a prospective observational study. BMJ Open 2019;9:e028810. doi:10.1136/ bmjopen-2018-028810

- Prepublication history for this paper is available online. To view these files, please visit the journal online (http://dx.doi. org/10.1136/bmjopen-2018028810).

Received 24 December 2018 Revised 27 April 2019 Accepted 29 May 2019
Check for updates

(C) Author(s) (or their employer(s)) 2019. Re-use permitted under CC BY-NC. No commercial re-use. See rights and permissions. Published by BMJ.

For numbered affiliations see end of article.

Correspondence to

Esmee Venema;

e.venema@erasmusmc.nl

\section{ABSTRACT}

Introduction The efficacy of both intravenous treatment (IVT) and endovascular treatment (EVT) for patients with acute ischaemic stroke strongly declines over time. Only a subset of patients with ischaemic stroke caused by an intracranial large vessel occlusion (LVO) in the anterior circulation can benefit from EVT. Several prehospital stroke scales were developed to identify patients that are likely to have an LV0, which could allow for direct transportation of EVT eligible patients to an endovascular-capable centre without delaying IVT for the other patients. We aim to prospectively validate these prehospital stroke scales simultaneously to assess their accuracy in predicting LVO in the prehospital setting.

Methods and analysis Prehospital triage of patients with suspected stroke symptoms (PRESTO) is a prospective multicentre observational cohort study in the southwest of the Netherlands including adult patients with suspected stroke in the ambulance. The paramedic will assess a combination of items from five prehospital stroke scales, without changing the normal workflow. Primary outcome is the clinical diagnosis of an acute ischaemic stroke with an intracranial LVO in the anterior circulation. Additional hospital data concerning the diagnosis and provided treatment will be collected by chart review. Logistic regression analysis will be performed, and performance of the prehospital stroke scales will be expressed as sensitivity, specificity and area under the receiver operator curve.

Ethics and dissemination The Institutional Review Board of the Erasmus MC University Medical Centre has reviewed the study protocol and confirmed that the Dutch Medical Research Involving Human Subjects Act (WMO) is not applicable. The findings of this study will be disseminated widely through peer-reviewed publications and conference presentations. The best performing scale, or the simplest scale in case of clinical equipoise, will be integrated in a decision model with other clinical characteristics and real-life driving times to improve prehospital triage of suspected stroke patients. Trial registration number NTR7595.

\section{INTRODUCTION}

Rapid treatment with intravenous treatment (IVT) is effective for patients with an

\section{Strengths and limitations of this study}

- Prospective simultaneous validation of several prehospital stroke scales allows for direct comparison of their accuracy.

- In contrast to previous studies based on in-hospital assessment by experienced physicians, assessment of the prehospital stroke scales will be performed by paramedics in daily clinical practice.

- The results of this study will provide unique insight in the characteristics of an unselected group of patients with suspected stroke in the prehospital setting.

- The best performing scale will be integrated in a prehospital decision tool with other clinical characteristics and real-life driving times to select those patients that benefit from direct transportation to an endovascular-capable centre.

- Performance will be measured with the area under the receiver operator curve, which does not always relate directly to the clinical usefulness of these scales.

ischaemic stroke of $<4.5$ hours after onset. ${ }^{12}$ However, the effect of IVT is limited for ischaemic stroke caused by an intracranial large vessel occlusion (LVO) in the anterior circulation, which accounts for approximately $30 \%$ of the patients. ${ }^{3}$ These patients can benefit from endovascular treatment (EVT), preferably started within 6 hours after onset of symptoms, but this treatment can only be performed in specialised intervention centres. ${ }^{4}$ The effect of both treatments strongly declines over time..$^{5-7}$ In current clinical practice, most suspected stroke patients are transported by ambulance to the nearest hospital for immediate treatment with IVT. Patients can subsequently be transferred to an endovascular capable centre, if eligible for EVT. This is one of the main causes of 
treatment delay and is associated with worse functional outcomes after EVT. ${ }^{89}$

Several prehospital stroke scales were developed to identify patients that are likely to have an LVO, which could allow for direct transportation of EVT eligible patients to an endovascular-capable centre without delaying IVT for the other patients. ${ }^{10}{ }^{11}$ Most of these scales were derived from the National Institute of Health Stroke Severity (NIHSS) score, and external validation was often attempted by retrospective assessment of the items based on the NIHSS score completed by the treating physician at the emergency department. ${ }^{12-14}$ The results of existing prehospital validation studies are limited due to small sample sizes, selected populations or the exclusion of stroke mimics. ${ }^{15-18}$ Further prospective validation is therefore required to assess and compare the accuracy of these scales when used by emergency medical services (EMS) personnel in a broad population of suspected stroke patients under circumstances that reflect usual care.

\section{Objective}

The primary objective of this study is to prospectively validate several prehospital stroke scales simultaneously to assess their accuracy in predicting the likelihood of ischaemic stroke caused by an intracranial LVO in the prehospital setting.

\section{METHODS AND ANALYSIS \\ Study design}

Prehospital triage of patients with suspected stroke symptoms (PRESTO) is a prospective multicentre observational cohort study. Patients will be recruited in the ambulance, and a combination of items from different prehospital stroke scales will be assessed by the paramedic. The normal workflow will not be affected, and there is no intervention. Additional hospital data will be collected by chart review. Routinely performed neuroimaging will be collected and centrally assessed. Follow-up will only be performed in patients with a final diagnosis of ischaemic stroke.

\section{Study population}

We will include patients in the southwest of the Netherlands, a region with approximately 2 million inhabitants. Participating paramedics have ample experience with the initial management of patients with acute neurological deficits, and they received additional training before the start of the study with regards to the study procedures and the use of the prehospital stroke scales. Additional to the prior training, an instruction video is available for all paramedics. Also, during the duration of the study, regular visits are paid to all ambulance stations to provide feedback and address uncertainty or questions of the paramedics. All adult patients with acute neurological deficit, defined as at least one point on the Face-ArmSpeech-Test (FAST), and a suspected diagnosis of stroke by the paramedic, will be included. Patients with a blood glucose level below $2.5 \mathrm{mmol} / \mathrm{L}$ will be excluded.

\section{Prehospital stroke scales}

We choose five well known prehospital stroke scales to validate: the Los Angeles Motor Scale (LAMS), ${ }^{19} 20$ the Rapid Arterial oCclusion Evaluation (RACE), ${ }^{18}$ the Cincinnati Stroke Triage Assessment Tool (C-STAT), ${ }^{21}$ the Prehospital Acute Stroke Severity scale (PASS) ${ }^{22}$ and the Gaze-Face-Arm-Speech-Test (G-FAST) ${ }^{23}$ These scales have many similarities in the items that are being used, but there are differences in the scoring systems and the degree of complexity of these scores. In the PRESTO study, we will assess a combination of the items used in these five scales (table 1 ).

\section{Data collection}

Eligible patients presenting with suspected stroke symptoms will be recruited in the ambulance. The items from the prehospital stroke scales will be assessed by the paramedic and entered in a web-based database (LimeSurvey $\mathrm{GmbH} /$ Carsten Schmitz, https://www.limesurvey.org). The paramedic will also enter the transportation number (to link with EMS data and hospital data), the time of symptom onset or last known well (according to patient or bystander), the side of the hemiparesis (if applicable) and the presence of a known neurological deficit on the symptomatic side. Data concerning demographics, vital functions, general neurological examination and transportation times will be collected from the EMS databases.

After arrival in the hospital, patients will receive the usual care. A non-contrast CT scan and additional imaging (eg, CT angiography (CTA), digital subtraction angiography (DSA) and/or CT perfusion) can be performed as part of the regular workup of a suspected stroke. No additional imaging will be performed in the context of this study. Clinical data concerning the medical history, medication use, laboratory results, physical examination and diagnosis will be collected by chart review. All diagnostic neuroimaging data and radiology reports will be collected. If applicable, we will also collect information on the given treatment and corresponding treatment times (eg, the door-to-needle time, the door-to-groin time, the imaging-to-treatment time and the door-in-door-out time of transferred patients).

Follow-up will only be collected for patients with a final diagnosis of acute ischaemic stroke. We will use the outcome registration of the hospitals to collect length of hospital stay, discharge destination and the modified Rankin Scale (mRS) score after 90 days.

\section{Outcome measures}

Primary outcome will be the clinical diagnosis of an acute ischaemic stroke with an intracranial LVO in the anterior circulation, defined as an occlusion of the internal carotid artery, the middle cerebral artery segment M1 or M2 or the anterior cerebral artery segment A1 or A2 (assessed on CTA or DSA). Secondary outcome measures 
Table 1 Overview of the items and corresponding scores used in the prehospital stroke scales

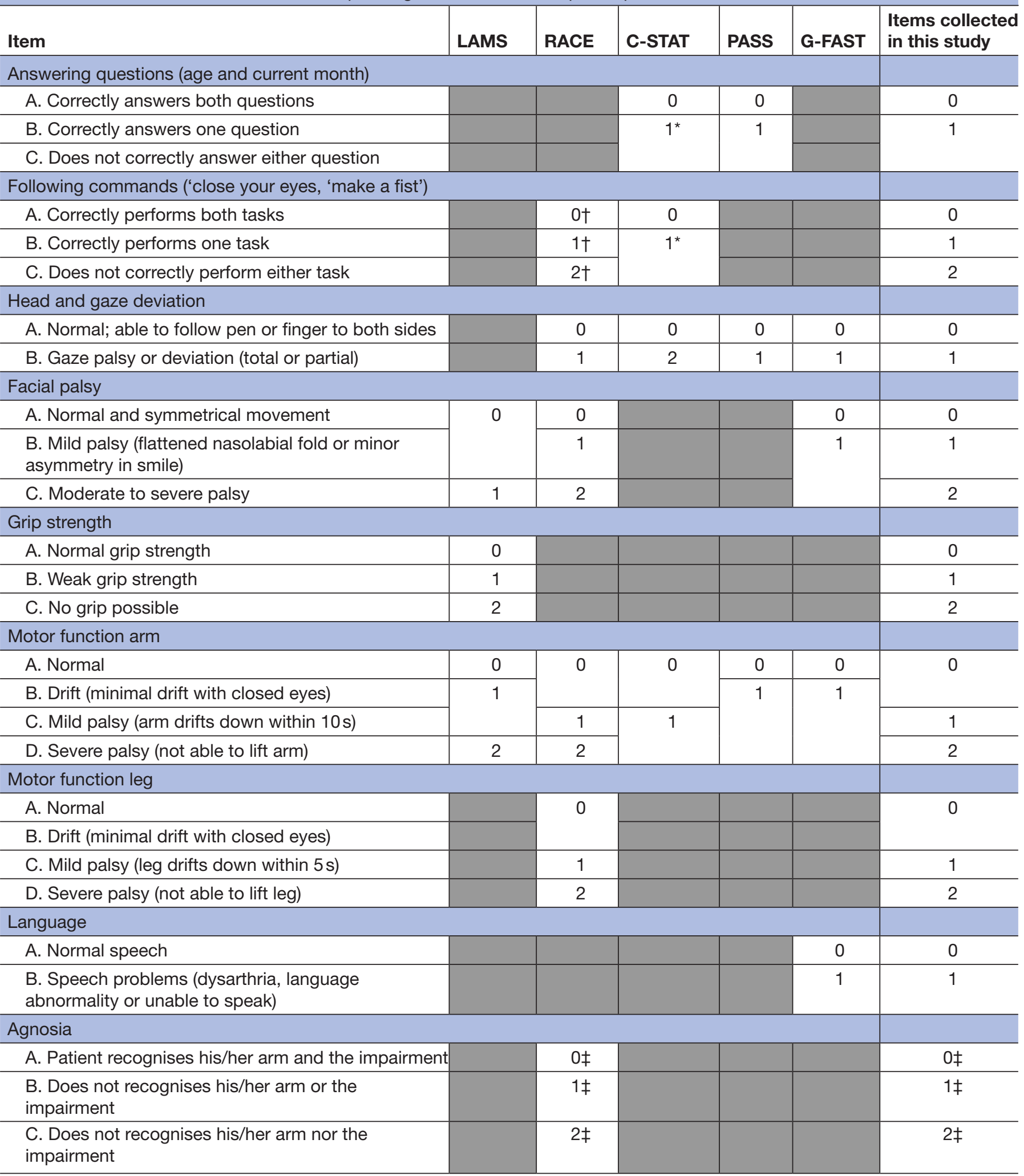

*Point if the patient answers at least one question incorrect and does not follow at least one command.

†Only scored if right hemiparesis $¥$ Only scored if left hemiparesis.

C-STAT, Cincinnati Stroke Triage Assessment Tool; G-FAST, Gaze-Face-Arm-Speech-Test; LAMS, Los Angeles Motor Scale; PASS,

Prehospital Acute Stroke Severity scale; RACE, Rapid Arterial oCclusion Evaluation. 
include the presence of an LVO in the posterior circulation (vertebral artery or basilar artery), the final diagnosis at hospital discharge, the given treatment (IVT, EVT or both) and corresponding treatment times and the functional outcome, measured with the 90-day mRS.

\section{Sample size calculation}

At least 100 events (ie, intracranial LVOs) are required for the external validation of predictive models. ${ }^{24}{ }^{25}$ The annual incidence of suspected ischaemic stroke within 6 hours after onset of symptoms is estimated to be 50 per 100000 people, based on an earlier cohort study. ${ }^{14}$ In the catchment area of the participating EMS (approximately 2 million inhabitants), this would imply 1000 patients every year presenting with stroke symptoms within the 6-hour time window. Of these 1000 patients, approximately $15 \%$ are assumed to have an ischaemic stroke due to an LVO, $31 \%$ an ischaemic stroke without the presence of an LVO, $9 \%$ a transient ischaemic attack (TIA), $10 \%$ an intracerebral haemorrhage and $35 \%$ a stroke mimic. ${ }^{14}$ To reach the required number of 100 stroke patients with an LVO, we will have to include at least (number of cases/ prevalence $=100 / 0.15) 667$ patients with stroke symptoms of $<6$ hours. To allow for a $5 \%$ loss of follow-up, we will aim for a sample size of 700 patients.

After inclusion of the first 500 patients, we will perform an interim analysis to calculate the percentage of LVO in our study population. If necessary, the required sample size will be adjusted based on this information. Although patients presenting after 6 hours will be included in the study, they will not count for the required sample size.

\section{Data analysis plan}

After completion of the last inclusion, the data will be checked, and the database will be locked for statistical analyses. We will report the absolute numbers and percentages of patients based on the final diagnosis (eg, ischaemic stroke, haemorrhagic stroke, TIA or stroke mimic) and, if applicable, the location of the intracranial LVO. For ischaemic stroke patients, we will report the given treatment (IVT, EVT or both) and corresponding treatment times, the number of interhospital transfers and the functional outcome after 90 days. Missing values will be imputed with simple imputation based on the mean or mode (if $<5 \%$ missing) or multiple imputations based on relevant covariates and outcome (if $>5 \%$ missing).

The different prehospital stroke scales will be reconstructed based on the items assessed in the ambulance (table 1). We will validate the prehospital stroke scales for patients presented within 6 hours after symptom onset using a logistic regression model with the presence of an $\mathrm{LVO}$ in the anterior circulation as outcome measure. We will analyse the scores both continuously and dichotomised, based on the previously reported cut points in the original studies. Sensitivity and specificity of all cut points will be reported separately. The global performance of the prehospital stroke scales will be expressed as the area under the receiver operator curve.
Prespecified sensitivity analyses will be performed for patients that presented more than 6 hours after symptom onset for the separate occlusion locations and the presence of an LVO in the posterior circulation. We will also assess the original outcome definitions as defined in each prehospital stroke scale instead of our own primary outcome, and we will analyse the correlation between the prehospital stroke scales and the NIHSS assessed at the emergency department. Additional analyses will be performed to predict the probability of treatment with EVT based on the prehospital stroke scales and relevant factors in the medical history, medication use or vital signs.

\section{Patient and public involvement}

Patients and public were not involved in the development of the research questions or the design of this study. All study participants and every interested person in public will have the possibility to read regular project updates on the project website (www.presto-studie.nl).

\section{Duration and current status of the study}

The study was registered in The Netherlands Trial Register on 11 November 2018 under number NTR7595 ( www.trialregister.nl). The study started on 13 August 2018 in the region Zuid-Holland Zuid and on 1 September 2018 in the region Rotterdam-Rijnmond. Recruitment of patients is ongoing, and at the time of submission, April 2019, 665 patients have been included in the study within 6 hours of symptom onset. In anticipation of a formal interim analysis, first raw data analysis shows a prevalence of $8 \%$ LVO in our study population. Based on this information, we increased our sample size to 1250 patients. With the current inclusion rate, we expect to reach the required sample size of 1250 patients by September 2019 .

\section{Ethics and dissemination}

Ethical aspects and informed consent

This study will be conducted in accordance with the principles of Good Clinical Practice, the Dutch Agreement on Medical Treatment Act (WGBO) and the European General Data Protection Regulation. The Institutional Review Board of the Erasmus MC University Medical Centre has reviewed the study protocol and confirmed that the Dutch Medical Research Involving Human Subjects Act (WMO) is not applicable.

Acquiring informed consent can be very challenging in the prehospital inclusion of suspected stroke patients. Many patients suffer from a language deficit, anosognosia, or other cognitive symptoms that impede an informed consent procedure, and often there is no (legal) representative of the patient present in the prehospital setting. Furthermore, an adequate informed consent procedure takes time, which is not available in the prehospital setting. Sometimes a deferred consent procedure can be used, but in the context of the WGBO, this should be done by the treating physician. Since our unselected population of patients, including many stroke mimics, 
will spread towards different directions after presentation in the hospital, a disproportionate number of healthcare providers from a variety of specialisms (eg, neurologists, emergency physicians, internists, cardiologists) should be involved in the research to enable a deferred consent procedure.

The extent of the effort by a large number of healthcare providers needed to obtain permission from the participating patients is disproportionate to the relatively limited sensitivity of the collected and linked personal data and the related limited intrusion to the personal privacy. We will therefore use an opt-out procedure in this study. The including paramedic will provide a leaflet with information about the study to the patient or their relatives. In this leaflet, we will explain that some routinely collected data can be collected from the EMS databases and the hospital charts for further analysis. Patients or their relatives are offered the opportunity to object to the use of these data in this study. When a patient or relative objects to study participation, all data will be destroyed, and the patient will be excluded from the study.

\section{Dissemination plan}

The main study results will be disseminated via publication in an international peer-reviewed journal and presentation at international conferences for stroke and emergency medicine experts. Representatives of the EMS providers and participating hospitals will be given the opportunity to comment on the manuscript and participate as coauthor, following the recommendations of the International Committee of Journal Editors. We plan to disseminate the results of the planned secondary analyses in one or more separate papers.

The best performing scale or the simplest scale in case of clinical equipoise, will be integrated in a decision model with other clinical characteristics and real-life driving times. ${ }^{26}$ This model can be implemented in an online tool to improve prehospital triage of patients with suspected stroke symptoms without harming those patients that benefit from rapid IVT in the nearest hospital. Patients eligible for EVT will be directly transported to an endovascular-capable centre, which will lead to an increased number of treated patients, reduced treatment times and improved patient outcomes. Moreover, avoiding unnecessary interhospital transfers will lead to more efficient use of EMS resources.

\section{Author affiliations \\ ${ }^{1}$ Public Health, Erasmus MC University Medical Centre, Rotterdam, The Netherlands ${ }^{2}$ Neurology, Erasmus MC University Medical Centre, Rotterdam, The Netherlands \\ ${ }^{3}$ Neurology, Albert Schweitzer Hospital, Dordrecht, The Netherlands \\ ${ }^{4}$ Neurology, Maasstad Hospital, Rotterdam, The Netherlands \\ ${ }^{5}$ Neurology, Franciscus Gasthuis \& Vlietland, Rotterdam, The Netherlands \\ ${ }^{6}$ Ambulance Service Rotterdam-Rijnmond, Barendrecht, The Netherlands \\ ${ }^{7}$ Radiology \& Nuclear Medicine, Erasmus MC University Medical Centre, Rotterdam, The Netherlands}

Collaborators PRESTO Investigators: Diederik WJ Dippel; Bob Roozenbeek; Aad van der Lugt; Hester F Lingsma; Henk Kerkhoff; Anouk D Rozeman; Walid Moudrous; Frederique H Vermeij; Esmee Venema; Martijne HC Duvekot; Cees L
Alblas; Laus JJM Mulder; Bruno JM van Moll; Lisette Maasland; Roeland PJ van Eijkelenburg; Marileen Biekart; Frits GJ Weijschede; Bianca Buijck; Amber Hoek; Jeanette Bakker; Erick Oskam; Rezan Demir; Mandy MA van der Zon; Egon D Zwets; Mirjam Woudenberg; Anja Noordam-Reijm; Timo Bevelander; Vicky Chalos; Eveline JA Wiegers.

Contributors EV contributed to study conception and design and wrote the manuscript. HFL, DWJD and BR contributed to study conception and design and critical review of the manuscript. MHCD, ADR, HK, WM, FHV, MB and AvdL contributed to study design and critical review of the manuscript.

Funding This study was supported by unrestricted grants from the BeterKeten collaboration and the Theia foundation (Zilveren Kruis). Both funding organisations were not involved in the design and conduct of the study.

Competing interests None declared.

Patient consent for publication Not required.

Provenance and peer review Not commissioned; externally peer reviewed.

Open access This is an open access article distributed in accordance with the Creative Commons Attribution Non Commercial (CC BY-NC 4.0) license, which permits others to distribute, remix, adapt, build upon this work non-commercially, and license their derivative works on different terms, provided the original work is properly cited, appropriate credit is given, any changes made indicated, and the use is non-commercial. See: http://creativecommons.org/licenses/by-nc/4.0/.

\section{REFERENCES}

1. National Institute of Neurological Disorders and Stroke rt-PA Stroke Study Group. Tissue plasminogen activator for acute ischemic stroke. N Engl J Med 1995;333:1581-8.

2. Hacke W, Kaste M, Bluhmki E, et al. Thrombolysis with alteplase 3 to 4.5 hours after acute ischemic stroke. N Engl J Med 2008;359:1317-29.

3. Lakomkin N, Dhamoon M, Carroll K, et al. Prevalence of large vessel occlusion in patients presenting with acute ischemic stroke: a 10year systematic review of the literature. J Neurointerv Surg 2019;11.

4. Goyal M, Menon BK, van Zwam WH, et al. Endovascular thrombectomy after large-vessel ischaemic stroke: a meta-analysis of individual patient data from five randomised trials. Lancet 2016;387:1723-31.

5. Emberson J, Lees KR, Lyden P, et al. Effect of treatment delay, age, and stroke severity on the effects of intravenous thrombolysis with alteplase for acute ischaemic stroke: a meta-analysis of individual patient data from randomised trials. Lancet 2014;384:1929-35.

6. Mulder M, Jansen IGH, Goldhoorn RB, et al. Time to endovascular treatment and outcome in Acute Ischemic Stroke: MR CLEAN Registry Results. Circulation 2018;138:232-40.

7. Saver JL, Goyal M, van der Lugt A, et al. Time to treatment with endovascular thrombectomy and outcomes from ischemic stroke: a meta-analysis. JAMA 2016;316:1279-88.

8. Venema E, Boodt N, Berkhemer OA, et al. Workflow and factors associated with delay in the delivery of intra-arterial treatment for acute ischemic stroke in the MR CLEAN trial. J Neurointerv Surg 2018;10:424-8.

9. Froehler MT, Saver JL, Zaidat OO, et al. Interhospital transfer before thrombectomy is associated with delayed treatment and worse outcome in the STRATIS Registry (Systematic Evaluation of Patients Treated With Neurothrombectomy Devices for Acute Ischemic Stroke). Circulation 2017;136:2311-21.

10. Smith EE, Kent DM, Bulsara KR, et al. Accuracy of prediction instruments for diagnosing large vessel occlusion in individuals with suspected stroke: a systematic review for the 2018 guidelines for the early management of patients with Acute Ischemic Stroke. Stroke 2018;49:e111-e22.

11. Krebs W, Sharkey-Toppen TP, Cheek F, et al. Prehospital stroke assessment for large vessel occlusions: a systematic review. Prehosp Emerg Care 2018;22:180-8.

12. Heldner MR, Hsieh K, Broeg-Morvay A, et al. Clinical prediction of large vessel occlusion in anterior circulation stroke: mission impossible? J Neurol 2016;263:1633-40.

13. Turc G, Maïer B, Naggara $O$, et al. Clinical scales do not reliably identify acute ischemic stroke patients with large-artery occlusion. Stroke 2016;47:1466-72.

14. Zhao H, Coote S, Pesavento L, et al. Large vessel occlusion scales increase delivery to endovascular centers without excessive harm from misclassifications. Stroke 2017;48:568-73. 
15. Kim JT, Chung PW, Starkman S, et al. Field validation of the los angeles motor scale as a tool for paramedic assessment of stroke severity. Stroke 2017;48:298-306.

16. McMullan JT, Katz B, Broderick J, et al. Prospective prehospital evaluation of the cincinnati stroke triage assessment tool. Prehosp Emerg Care 2017;21:481-8.

17. Noorian AR, Sanossian N, Shkirkova K, et al. Los Angeles motor scale to identify large vessel occlusion: prehospital validation and comparison with other screens. Stroke 2018;49:565-72.

18. Pérez de la Ossa N, Carrera D, Gorchs M, et al. Design and validation of a prehospital stroke scale to predict large arterial occlusion: the rapid arterial occlusion evaluation scale. Stroke 2014;45:87-91.

19. Llanes JN, Kidwell CS, Starkman S, et al. The Los Angeles Motor Scale (LAMS): a new measure to characterize stroke severity in the field. Prehosp Emerg Care 2004;8:46-50.

20. Nazliel B, Starkman S, Liebeskind DS, et al. A brief prehospital stroke severity scale identifies ischemic stroke patients harboring persisting large arterial occlusions. Stroke 2008;39:2264-7.
21. Katz BS, McMullan JT, Sucharew $\mathrm{H}$, et al. Design and validation of a prehospital scale to predict stroke severity: Cincinnati Prehospital Stroke Severity Scale. Stroke 2015;46:1508-12.

22. Hastrup S, Damgaard D, Johnsen SP, et al. Prehospital acute stroke severity scale to predict large artery occlusion: design and comparison with other scales. Stroke 2016;47:1772-6.

23. Scheitz JF, Abdul-Rahim AH, Maclsaac RL, et al. Clinical Selection Strategies to Identify Ischemic Stroke Patients With Large Anterior Vessel Occlusion: Results From SITS-ISTR (Safe Implementation of Thrombolysis in Stroke International Stroke Thrombolysis Registry). Stroke 2017;48:290-7.

24. Vergouwe Y, Steyerberg EW, Eijkemans MJ, et al. Substantial effective sample sizes were required for external validation studies of predictive logistic regression models. $J$ Clin Epidemiol 2005;58:475-83.

25. Collins GS, Ogundimu EO, Altman DG. Sample size considerations for the external validation of a multivariable prognostic model: a resampling study. Stat Med 2016;35:214-26.

26. Venema E, Lingsma HF, Chalos V, et al. Personalized prehospital triage in acute ischemic stroke. Stroke 2019;50:313-20. 